\title{
Early Integrated Telehealth versus In-Person Palliative Care for Patients with Advanced Lung Cancer: A Study Protocol
}

\author{
Isaac S. Chua, MD, ${ }^{1, *}$ Finly Zachariah, MD, ${ }^{2, *}$ William Dale, $M D^{2},{ }^{2}$ Josephine Feliciano, MD, ${ }^{3}$ \\ Laura Hanson, MD, ${ }^{4}$ Leslie Blackhall, MD ${ }^{5}$ Tammie Quest, MD, ${ }^{6}$ Kimberly Curseen, MD, \\ Carl Grey, MD, ${ }^{7}$ Ramona Rhodes, MD, ${ }^{8}$ Laura Shoemaker, DO, ${ }^{9}$ Maria Silveira, MD, ${ }^{10}$ \\ Stacy Fischer, MD, ${ }^{11}$ Sean O'Mahony, MD, ${ }^{12}$ Kostantinos Leventakos, MD, ${ }^{13}$ Chardria Trotter, MBA, MPH, ${ }^{14}$ \\ Isabella Sereno, MEd, ${ }^{14}$ Mihir Kamdar, MD, ${ }^{14}$ Jennifer Temel, MD, ${ }^{14}$ and Joseph A. Greer, $\mathrm{PhD}^{14}$
}

\begin{abstract}
Introduction: Early palliative care (PC) integrated with oncology care improves quality of life (QOL), depression symptoms, illness understanding, and end-of-life (EOL) care for patients with advanced lung cancer. The aims of this trial are to compare the effect of delivering early integrated PC through telehealth versus in-person on patient and caregiver outcomes. We hypothesize that both modalities for delivering early PC would be equivalent for improving patient QOL, communication about EOL care preferences with their oncologist, and length of stay in hospice.

Methods: For this comparative effectiveness trial, we will enroll and randomize 1250 adult patients with advanced nonsmall cell lung cancer (NSCLC), who are not being treated with curative intent, to receive either early integrated telehealth or in-person PC at 20 cancer centers throughout the United States. Patients may also invite a family caregiver to participate in the study. Patients and their caregivers in both study groups meet at least every four weeks with a PC clinician from within 12 weeks of patient diagnosis of advanced NSCLC until death. Participants complete measures of QOL, mood, and quality of communication with oncologists at baseline before randomization and at 12, 24,36, and 48 weeks. Information on health care utilization, including length of stay in hospice, will be collected from patients' health records. To test equivalence in outcomes between study groups, we will compute analysis of covariance and mixed linear models, controlling for baseline scores and study site. Study Implementation and Stakeholder Engagement: To ensure that this comparative effectiveness trial and findings are as patient centered and meaningful as possible, we have incorporated a robust patient and stakeholder engagement plan. Our stakeholder partners include (1) patients/families, (2) PC clinicians, (3) telehealth experts and clinician users, (4) representatives from health care systems and medical insurance providers, and (5) health care policy makers and advocates. These stakeholders will inform and provide feedback about every phase of study implementation.
\end{abstract}

Keywords: early palliative care; lung cancer; telehealth

${ }^{1}$ Dana-Farber Cancer Institute, Boston, Massachusetts.

${ }^{2}$ City of Hope, Duarte, California.

${ }^{3}$ The Johns Hopkins University School of Medicine, Baltimore, Maryland.

${ }^{4}$ University of North Carolina School of Medicine, Chapel Hill, North Carolina.

${ }^{5}$ University of Virginia, Charlottesville, Virginia.

${ }^{6}$ Emory University, Atlanta, Georgia.

${ }^{7}$ Wake Forest Baptist Health, Winston-Salem, North Carolina.

${ }^{8}$ University of Texas Southwestern Medical Center, Dallas, Texas.

${ }^{9}$ Cleveland Clinic, Cleveland, Ohio.

${ }^{10}$ Michigan Medicine, Ann Arbor, Michigan.

${ }^{11}$ University of Colorado Hospital, Aurora, Colorado.

${ }^{12}$ Rush University Medical Center, Chicago, Illinois.

${ }^{13}$ Mayo Clinic, Rochester, Minnesota.

${ }^{14}$ Massachusetts General Hospital, Boston, Massachusetts.

*These authors are co-first authors for this article.

Accepted July 2, 2019. 


\section{Introduction}

\section{Background}

$\mathbf{E}$ ARLY PALliative CARE (PC) integrated with oncology care in the outpatient setting improves quality of life (QOL), mood, illness understanding, and end-of-life (EOL) care among patients with advanced cancer and their caregivers. ${ }^{1-5}$ Therefore, early integrated $\mathrm{PC}$ has become the standard of care for patients with advanced cancer. ${ }^{6}$ However, a shortage of PC professionals ${ }^{7}$ and lack of capacity within cancer clinics 8,9 are major barriers to wide-scale implementation of early integrated PC. Novel models of care delivery, such as telehealth videoconferencing, have the potential to increase access to and efficient utilization of limited $\mathrm{PC}$ resources in a patient-centered manner.

Telehealth is the delivery and facilitation of healthrelated services, including direct medical care, provider and patient education, health information services, and self-care through telecommunications and digital communication technologies. ${ }^{10}$ Studies show that telehealth not only increases access to health care services, especially where the availability of specialty clinicians is limited, but also is equivalent to in-person services for mental health, rehabilitation, and dermatology. ${ }^{11,12}$ Although researchers are beginning to study the use of videoconferencing to conduct virtual home visits in the context of providing PC, ${ }^{13,14}$ prior studies have demonstrated that telephone-based psychoeducational interventions, delivered by PC-trained advanced practice nurses, improve QOL and lower depression symptoms among patients with newly diagnosed advanced cancers as well as decrease depression symptoms in their caregivers. ${ }^{15,16}$ Telehealth may be especially helpful for caregivers by improving access to PC for patients' loved ones, enhancing communication between multiple family members and the care team, and reducing the burden of attending in-person clinic visits. ${ }^{14}$

In this study, we will adapt the evidence-based early integrated in-person PC model to a telehealth platform using secure videoconferencing, thereby enabling PC clinicians to provide virtual home visits and increase access to specialty services in a patient-centered manner. If the proposed study demonstrates that telehealth is as effective as (or superior to) in-person PC, such findings would address a major evidence gap between the data supporting early integrated in-person PC and lack of data regarding how to disseminate this care model most efficiently, equitably, and effectively. We, therefore, present the study protocol to detail the methods and procedures for implementing this trial and to facilitate access and dissemination in delivering the early integrated PC model either in-person or through telehealth.

\section{Objectives and hypotheses}

The objectives of this study are to examine the effects of early integrated telehealth PC versus in-person PC on patients' QOL, patient-clinician communication about EOL care preferences, patient length of stay in hospice, caregiver participation in PC visits, and patient and caregiver satisfaction with care. We hypothesize the following:

(1) Patients assigned to early integrated telehealth PC will report QOL that is equivalent to patients receiving in-person PC.
(2) Early integrated telehealth PC will be equivalent to in-person PC with respect to the rate by which patients communicate their EOL care preferences with their clinicians and length of stay in hospice.

(3) Caregivers who receive early integrated telehealth PC will participate in a higher percentage of visits with the PC clinician as well as report better QOL and lower depression symptoms compared with those receiving in-person $\mathrm{PC}$.

(4) Patients and caregivers will report greater satisfaction with early integrated telehealth PC compared with in-person PC.

\section{Methods}

\section{Study design and setting}

This study is a multicenter randomized comparative effectiveness trial of early integrated telehealth versus inperson PC. We will recruit 1250 patients with advanced nonsmall cell lung cancer (NSCLC) and up to 1250 of their caregivers through 20 Palliative Care Research Cooperative (PCRC) designated institutions across the United States. The Massachusetts General Hospital (MGH) is the lead site for the study and the additional 19 PCRC institutions. Figure 1 details the study design.

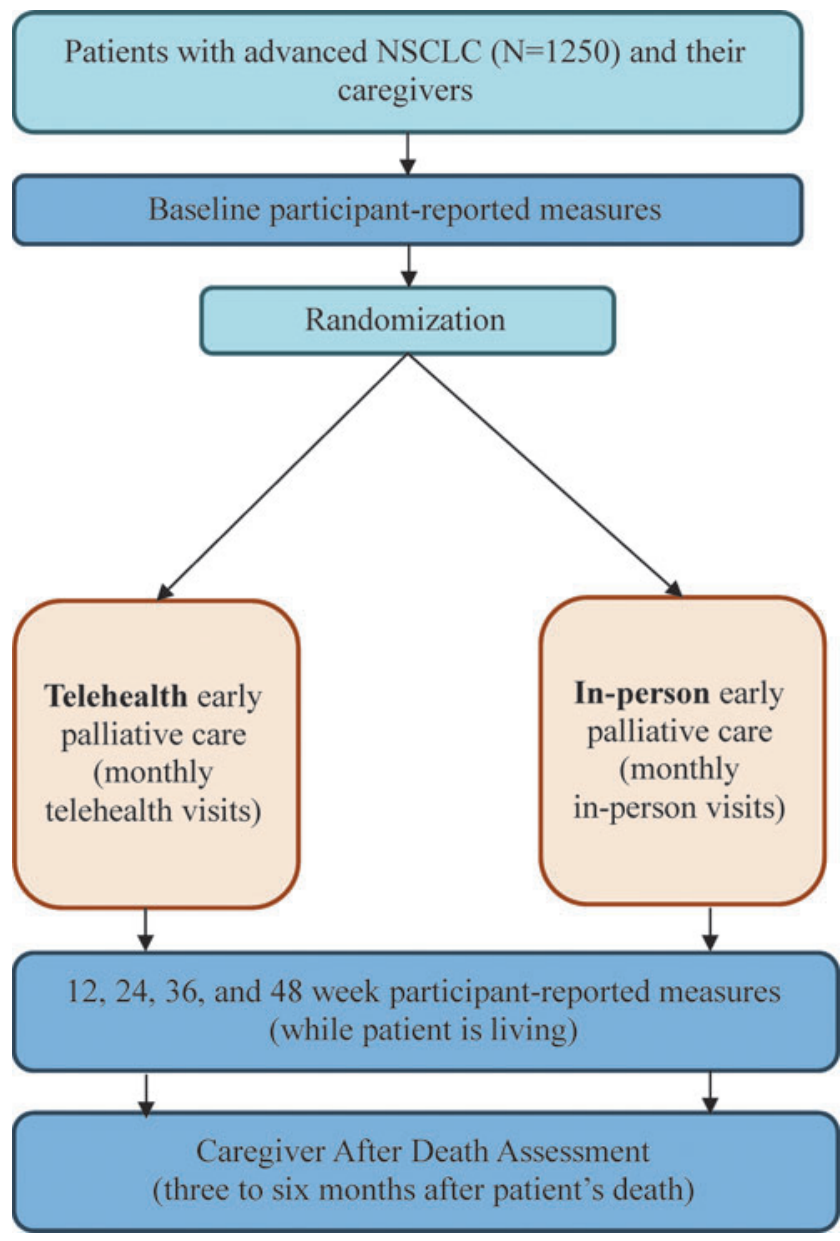

FIG. 1. Study design. NSCLC, nonsmall cell lung cancer. 


\section{Early integrated PC intervention}

The PC interventionists must be specialty-trained experienced or board-certified PC physicians or advanced practice nurses who provide care in the outpatient oncology setting. The Early PC Treatment Guide for this trial is based on our prior studies that detail the elements of this care model. ${ }^{17,18}$ Specifically, the practice of early integrated PC includes six domains: (1) developing and maintaining the therapeutic relationship with patients and caregivers, (2) assessing and treating patient symptoms, (3) providing support and reinforcement of coping with advanced cancer in patients and caregivers, (4) assessing and enhancing prognostic awareness and illness understanding in patients and caregivers, (5) assisting with treatment decision making, and (6) planning for EOL care. Table 1 includes an outline of these Early PC domains.

\section{Study arms}

Enrolled patients and caregivers will be randomized to either early integrated telehealth PC or in-person PC. The initial PC visit in both groups occurs in person within four weeks of enrollment in the outpatient clinic setting, with subsequent visits taking place every four weeks (either through telehealth or in person depending on group assignment). The purpose of the first in-person visit in the telehealth group is to establish rapport between the PC clinician and the patient and family before transitioning to videoconference visits. Patients who survive greater than 18 months will be permitted to decrease the frequency of PC visits per their preference. If participants in either group miss their scheduled visit, and it cannot be rescheduled within four weeks of their prior visit, the PC clinician may conduct the visit through telephone within seven days from the missed visit. Patients scheduled to meet with PC clinicians who do not speak their native language will be seen in conjunction with a hospital interpreter (either in person or through videoconferencing). After each study visit, PC clinicians document the encounter in the patient's health record. PC clinicians also complete an electronic survey to note the topics addressed during each study visit and whether a caregiver was present.

Telehealth PC arm. For the telehealth group, the research team contacts patients within three business days of enrollment to inquire whether they have videoconference capability (i.e., device with a camera, speaker, microphone, and sufficient Internet connection). Any patients who do not have equipment, or whose equipment is insufficient for the videoconference platform, will be sent a cellular-enabled study-supported iPad secured only for videoconferencing. The research team then conducts a videoconference test call with the patient and caregivers before the first telehealth PC visit. Caregivers at other locations from the patient wishing to join telehealth visits are required to use their own video and cellular service. The research team uses Vidyo videoconference platform and skills-based routing (SBR) patient management software for the delivery of telehealth. To maintain privacy, Vidyo ensures that the video and audio transmissions are encrypted and allows users access to these transmissions through secure virtual video rooms, which are managed by SBR.

After the initial in-person visit, subsequent visits take place in patients' homes (or the setting of their choice) through telehealth at least every four weeks until death. PC clinicians may see patients more frequently than every four weeks, at their discretion. If technical problems occur with the telehealth technology during a patient's scheduled virtual visit, then the PC clinician conducts the remainder of the visit through telephone. Patients may be scheduled to meet with the PC clinician in the clinic if requested by the patient or a clinician. If a patient has an in-person visit with the PC clinician, they will still be scheduled for their telehealth visits every four weeks. The PC clinician is required to communicate with the attending oncology clinicians through e-mail, telephone, or in person after each patient encounter.

In-Person PC arm. Patients randomized to in-person PC are scheduled for their first PC visit within four weeks of enrollment and then at least every four weeks thereafter until the patient is no longer able to come into the clinic or death. $\mathrm{PC}$ visits are scheduled on the same day as oncology visits unless the patient is agreeable to scheduling the $\mathrm{PC}$ visit on a different day. Joint visits with PC and oncology (i.e., both clinicians seeing the patient together in one visit) are recommended but not required. PC clinicians may see patients more often than every four weeks at their discretion. If patients do not have a scheduled visit to the cancer center, the PC clinician will contact them through telephone within four weeks of their prior appointment to conduct the visit.

\section{Randomization}

Patients from the 20 participating sites will be entered on the study centrally by the MGH research team. The research staff from the participating institutions will confirm eligibility criteria and fax or e-mail the following documents to the MGH research team: deidentified signed consent form/s, copy of baseline assessment, and a completed eligibility checklist. The MGH research team follows the Dana-Farber/ Harvard Cancer Center Standard Operating Procedure for Human Subject Research (Subject Protocol Registration) and registers the participant on the protocol. Once the patient has been registered, a member of the MGH research team (independent from study staff who recruit, enroll, and administer assessments to participants) will perform randomization procedures using on a computer-generated block randomization schema, stratified by study site.

\section{Outcome measures}

The study outcome measures include a combination of participant self-report questionnaires and data from patients' electronic health records. See Table 2 for a description of the study self-report measures.

Primary outcome. Patient QOL at 24 weeks: Functional Assessment of Cancer Therapy-Lung (FACT-L) ${ }^{19}$

\section{Secondary outcomes}

- Patient-clinician communication about EOL preferences: Patient Prognosis and Treatment Perceptions Questionnaire (PTPQ) ${ }^{20,21}$

- Patient length of stay in hospice: Days from hospice referral until death per electronic health record review 
Table 1. Outline of Early Palliative Care Intervention Guide

\begin{tabular}{|c|c|c|c|}
\hline Visit timing & Domain & Elements & Key points \\
\hline Initial visits & $\begin{array}{l}\text { Therapeutic } \\
\text { relationship }\end{array}$ & $\begin{array}{l}\text { - Introducing PC } \\
\text { - Understanding the } \\
\text { patient and caregiver } \\
\text { experience } \\
\text { - Building trust with the } \\
\text { patient and caregiver }\end{array}$ & $\begin{array}{l}\text { - Develop a strong therapeutic relationship with patients } \\
\text { and caregivers } \\
\text { - Learn about the values, life goals, and experiences of } \\
\text { patients and their caregivers both before and after the } \\
\text { cancer diagnosis } \\
\text { - Develop trust and credibility with patients and } \\
\text { caregivers by providing reassurance and outlining } \\
\text { parameters of communication }\end{array}$ \\
\hline \multirow[t]{2}{*}{$\begin{array}{l}\text { Visits } \\
\text { throughout } \\
\text { the course } \\
\text { of illness }\end{array}$} & Patient symptoms & $\begin{array}{l}\text { - Assessing and treating } \\
\text { symptoms } \\
\text { - Coordinating symptom } \\
\text { management with } \\
\text { oncology } \\
\text { - Providing referral for } \\
\text { symptom management }\end{array}$ & $\begin{array}{l}\text { - Clarify the symptoms the patient will likely experience } \\
\text { and offer reassurance about the methods for reporting } \\
\text { and treating symptoms } \\
\text { - At every visit, elicit existing and new symptom } \\
\text { concerns } \\
\text { - Maintain ongoing effective communication with } \\
\text { oncologists to define mutual collaboration and work } \\
\text { within their preferred practice patterns } \\
\text { - Emphasize team approach to care by referring to } \\
\text { specialty care, mental health, alternative medicine, and } \\
\text { spiritual support as needed }\end{array}$ \\
\hline & $\begin{array}{l}\text { Coping with } \\
\text { advanced } \\
\text { cancer }\end{array}$ & $\begin{array}{l}\text { - Reviewing and validating } \\
\text { prior coping efforts } \\
\text { - Discussing and } \\
\text { advocating for different } \\
\text { methods of coping } \\
\text { - Supporting caregiver } \\
\text { coping } \\
\text { - Providing referral for } \\
\text { additional support }\end{array}$ & $\begin{array}{l}\text { - Recognize that patients and caregivers bring their own } \\
\text { expertise in coping to the current circumstance based } \\
\text { on prior experiences } \\
\text { - Introduce strategies to help improve adjustment and } \\
\text { meaning in life (e.g., behavioral, cognitive, and } \\
\text { spiritual approaches; accepting illness while } \\
\text { maintaining hope; social support) } \\
\text { - Bolster caregiver coping by assessing burden, } \\
\text { enhancing their communication with patients, and } \\
\text { recommending additional support } \\
\text { - Involve other members of the team for patients and } \\
\text { caregivers who may be experiencing severe distress } \\
\text { (e.g., social work, psychology) }\end{array}$ \\
\hline \multirow[t]{2}{*}{$\begin{array}{l}\text { Visits during } \\
\text { clinical } \\
\text { turning } \\
\text { points }\end{array}$} & $\begin{array}{l}\text { Prognostic } \\
\text { awareness and } \\
\text { illness } \\
\text { understanding }\end{array}$ & $\begin{array}{l}\text { - Communicating with } \\
\text { oncology } \\
\text { - Exploring goals and } \\
\text { values } \\
\text { - Assessing and informing } \\
\text { patient expectation of } \\
\text { prognosis } \\
\text { - Conducting } \\
\text { conversations with } \\
\text { caregivers }\end{array}$ & $\begin{array}{l}\text { - Consult with the oncologist to ensure the care team is } \\
\text { consistent with their understanding of the patient's } \\
\text { prognosis } \\
\text { - Assess patient's hopes and expectations for treatment } \\
\text { and future to clarify the patient's level of prognostic } \\
\text { awareness } \\
\text { - Recognize that illness understanding often vacillates } \\
\text { between more and less realistic expectations and work } \\
\text { to improve prognostic awareness } \\
\text { - Include both patients and caregivers in conversations } \\
\text { about prognosis and illness understanding when } \\
\text { possible }\end{array}$ \\
\hline & $\begin{array}{c}\text { Treatment } \\
\text { decision } \\
\text { making }\end{array}$ & $\begin{array}{l}\text { - Assessing patient values } \\
\text { in treatment decision } \\
\text { making } \\
\text { - Discussing treatment } \\
\text { considerations } \\
\text { - Supporting treatment } \\
\text { decisions }\end{array}$ & $\begin{array}{l}\text { - Elicit information from patients and caregivers } \\
\text { regarding their decision-making style, quality versus } \\
\text { quantity of life concerns, and life goals } \\
\text { - Provide support for patients and caregivers to } \\
\text { understand the efficacy, and risks and benefits } \\
\text { associated with cancer treatment } \\
\text { - Clarify any misunderstanding about treatment, support } \\
\text { patient decision making and freedom to change course }\end{array}$ \\
\hline $\begin{array}{l}\text { Visits near } \\
\text { EOL }\end{array}$ & EOL care & $\begin{array}{l}\text { - Discussing EOL care } \\
\text { options } \\
\text { - Supporting caregivers in } \\
\text { EOL care coordination } \\
\text { and bereavement }\end{array}$ & $\begin{array}{l}\text { - Discuss/review selection of health care proxy, } \\
\text { determination of resuscitation preferences, transition to } \\
\text { hospice care, and location of death } \\
\text { - Determine available resources for EOL care and } \\
\text { whether it is appropriate for patients to receive care in } \\
\text { the home or other settings, and provide resources and } \\
\text { counseling for bereavement for caregivers }\end{array}$ \\
\hline
\end{tabular}

EOL, end-of-life; PC, palliative care. 
Table 2. Study Participant-Reported Measures

\begin{tabular}{|c|c|c|c|}
\hline Name of measure & Description & Participant & Time points \\
\hline $\begin{array}{l}\text { Sociodemographic } \\
\text { Questionnaire }\end{array}$ & $\begin{array}{l}\text { 19-Item measure on age, gender, race/ } \\
\text { ethnicity, marital status, religion, education } \\
\text { level, computer experience, and travel time } \\
\text { and transportation mode to the cancer center }\end{array}$ & Patient and caregiver & Baseline only \\
\hline $\mathrm{SCQ}^{28}$ & $\begin{array}{l}\text { 16-Item instrument assessing comorbidities } \\
\text { such as heart disease, lung disease, diabetes } \\
\text { and arthritis. }\end{array}$ & Patient only & Baseline only \\
\hline FACT-L ${ }^{19}$ & $\begin{array}{l}\text { 35-Item QOL measure of physical, social, } \\
\text { emotional, and functional well-being ( } 27 \\
\text { items), as well as lung cancer symptoms ( } 8 \\
\text { items) over the past week }\end{array}$ & Patient only & All \\
\hline Patient PTPQ ${ }^{20,21}$ & $\begin{array}{l}\text { 13-Item measure of patients' illness } \\
\text { understanding, communication about } \\
\text { prognosis with oncologist, and discussions } \\
\text { and preferences regarding EOL care }\end{array}$ & Patient only & All \\
\hline PHQ-9 ${ }^{27}$ & $\begin{array}{l}\text { 9-Item measure of symptoms of major } \\
\text { depressive disorder in the past two weeks } \\
\text { per the criteria of the Diagnostic and } \\
\text { Statistical Manual of Mental Disorders }\end{array}$ & Patient only & All \\
\hline Brief Cope ${ }^{23,24}$ & $\begin{array}{l}\text { 28-Item questionnaire that assesses } 14 \\
\text { methods of coping (e.g., active, acceptance, } \\
\text { denial) using a 4-point Likert scale ranging } \\
\text { from } 1 \text { "a lot" to } 4 \text { "never" }\end{array}$ & Patient only & All \\
\hline Support Service Utilization & $\begin{array}{l}\text { Single-item measure of mental health care } \\
\text { utilization since diagnosis }\end{array}$ & Patient only & 24 weeks \\
\hline $\mathrm{HADS}^{26}$ & $\begin{array}{l}\text { 14-Item questionnaire that contains two 7-item } \\
\text { subscales assessing depression and anxiety } \\
\text { symptoms during the past week }\end{array}$ & Patient and caregiver & All \\
\hline $\begin{array}{l}\text { Satisfaction with Care } \\
\text { Delivery Questionnaire }^{22}\end{array}$ & $\begin{array}{l}\text { 13-Item measure of satisfaction with PC visits } \\
\text { with respect to: (1) convenience, (2) quality } \\
\text { of communication and personal connection, } \\
\text { (3) engagement of caregivers, and (4) } \\
\text { overall satisfaction }\end{array}$ & Patient and caregiver & $\begin{array}{l}12,24,36, \text { and } \\
48 \text { weeks }\end{array}$ \\
\hline CARGOQOL $^{25}$ & $\begin{array}{l}\text { 29-Item instrument measuring caregiver QOL } \\
\text { in multiple domains }\end{array}$ & Caregiver only & All \\
\hline Caregiver $\mathrm{PTPQ}^{20,21}$ & $\begin{array}{l}\text { 6-Item questionnaire adapted from the patient } \\
\text { PTPQ to assess caregivers' understanding of } \\
\text { their loved ones' illness understanding and } \\
\text { perceptions of communication about } \\
\text { prognosis, goals of care, and EOL care } \\
\text { preferences }\end{array}$ & Caregiver only & All \\
\hline After Death Assessment ${ }^{20}$ & $\begin{array}{l}\text { 3-Item measure in which caregivers rate the } \\
\text { care they received in the last week of } \\
\text { patient's life using a 10-point scale for (1) } \\
\text { quality of patient's death (ranging from } \\
\text { "worse possible" to "best possible), (2) } \\
\text { patient's physical distress (ranging from } \\
\text { "none" to "extremely distressed"), and (3) } \\
\text { patient's psychological distress (ranging } \\
\text { from "none" to "extremely upset") }\end{array}$ & Caregiver only & $\begin{array}{l}3 \text { months after } \\
\text { patient } \\
\text { death }\end{array}$ \\
\hline
\end{tabular}

CARGOQOL, Caregiver Oncology QOL Questionnaire; FACT-L, Functional Assessment of Cancer Therapy-Lung; HADS, Hospital Anxiety and Depression Scale; PHQ-9, Patient Health Questionnaire-9; PTPQ, Patient Prognosis and Treatment Perceptions Questionnaire; QOL, quality of life; SCQ, Self-Administered Comorbidity Questionnaire.

- Caregiver participation in PC visits: Rate of caregiver attendance in PC visits per PC clinician documentation of postvisit electronic survey

- Patient and caregiver satisfaction with care: Satisfaction with Care Delivery Questionnaire 22

- Patient QOL at 48 weeks: FACT-L. ${ }^{19}$

\section{Exploratory outcomes}

- Patient coping strategies: Brief COPE Questionnaire ${ }^{23,24}$

- Patient and caregiver prognostic understanding: Prognosis and Treatment Perceptions Questionnaire (PTPQ) ${ }^{20,21}$ 
- Caregiver QOL: Caregiver Oncology QOL Questionnaire (CARGOQOL) ${ }^{25}$

- Caregiver mood: Hospital Anxiety and Depression Scale (HADS) ${ }^{26}$

- Patient mood and depression symptoms: HADS ${ }^{26} ; \mathrm{Pa}-$ tient Health Questionnaire-9 (PHQ-9) ${ }^{27}$

- Patient health care utilization: PC visits, chemotherapy and radiation administration, emergency department visits, ICU admissions, hospitalizations, and location of death per electronic health record

- Caregiver perceptions of patient death: After Death Assessment. $^{20}$

\section{Covariates}

- Patient and caregiver sociodemographic data: Sociodemographic Questionnaire

- Patient clinical characteristics: Tumor type, cancer stage, date of diagnosis, performance status, etc. per electronic health record review

- Patient medical comorbidity: Self-Administered Comorbidity Questionnaire (SCQ) ${ }^{28}$

- Patient mental health service utilization: Support Service Utilization Questionnaire.

\section{Recruitment and study procedures}

Patient eligibility criteria. Eligible patients include adults (age $>18$ years old) diagnosed with advanced NSCLC within the prior 12 weeks who are being treated with noncurative (i.e., palliative) intent. The original protocol specified an 8-week window from diagnosis for eligibility, which we amended to 12 weeks to increase enrollment. The patient also must have an Eastern Cooperative Oncology Group performance status between 0 and 3, be able to read and respond to questions in English or Spanish, live in the state where the institutions' PC clinicians are licensed to practice, and receive primary cancer care at one of the participating sites. Patients are excluded if they are already receiving outpatient PC or hospice services or have cognitive or psychiatric conditions that prohibit study consent or participation, as determined by the treating oncologist.

Caregiver eligibility criteria. An eligible caregiver is an adult relative or friend ( $>18$ years old) who is identified by the patient participant and lives with the patient or has contact with them at least twice per week. The caregiver must also be able to read and respond to questions in English or Spanish. Caregivers are excluded if they have cognitive or psychiatric conditions that prohibit study consent or participation, as determined by the treating oncologist.

Recruitment. We are using the same recruitment and enrollment procedures as in our previous and ongoing trials. ${ }^{3,4}$ Before the study start, site investigators meet with their respective thoracic oncology teams to review recruitment and enrollment procedures. The research team then sends an email to the oncology clinicians to notify them when their patients appear to be eligible for the study.

If the oncology clinician deems that the patients are eligible, willing participants receive a detailed Health Insurance Portability and Accountability Act (HIPAA)-compliant consent form and are given the opportunity to sign written informed consent either with a clinician or with a research team member. Patients are then asked to complete baseline questionnaires on the day of enrollment. After completing the baseline questionnaires, patients are registered for the study and randomized. If patients do not complete the baseline questionnaires within two weeks of signing informed consent, they are not permitted to enroll in the study. Patients without available caregivers are still eligible to participate.

For patients who primarily or solely speak Spanish, a research staff member explains all study procedures and information regarding risks, benefits, and study contacts through the use of an interpreter or family member. Spanishspeaking participants may receive the institutional Spanish consent form for signing as well as a copy of the English consent form. Spanish-speaking participants are then provided with Spanish-version baseline questionnaires.

The research team also collects data from each enrolled patient about whether they have an eligible caregiver who may want to participate. As caregivers may not be present at every clinic appointment, they are eligible to consent for the study from the same day that the patient is registered and up to four weeks thereafter. Caregivers are asked to complete the baseline study questionnaires on the day of their enrollment. The clinician or research team member also reviews the study procedures and consent form with caregivers and obtains written informed consent. If caregivers do not complete the baseline questionnaires within two weeks of signing informed consent, they are not permitted to enroll in the study.

\section{Data collection and analysis plan}

Data collection: Surveys/medical records/administrative data. The research team administers participant selfreport assessments at baseline, and every 12 weeks from the date the patient baseline assessment was completed for up to 48 weeks (with a 2-week window to accommodate patient schedules). The team collects measures during regularly scheduled visits whenever possible. Participants complete assessments using Research Electronic Data Capture (REDCap), a free secure HIPAA-compliant web-based application hosted by the Partners HealthCare Research Computing, Enterprise Research Infrastructure \& Services group. Participants may also complete the assessments on paper or verbally over the telephone if necessary. Enrolled patients who are referred to hospice services while on study are not asked to complete study assessments, although they may continue to participate in PC visits either in person or through telehealth. The research team contacts caregivers approximately three months after the patient's death to complete the After Death Assessment. In addition, the research team reviews patients' health records at baseline to collect data on clinical characteristics, such as tumor type, and after the patient dies (or at 18 months from the date the last patient enrolls on the study) to collect information on health care utilization.

After each study visit, PC clinicians enter data on the topics addressed during the encounter with a patient using a REDCap survey. The domains included in the REDCap survey correspond to those in the Early PC Treatment Guide (Table 1) to allow the PC clinician to document the content areas addressed during the visit, including any referrals made 
or medications prescribed, and whether a caregiver was present. The PC clinician also documents the type of visit (in person, telehealth, or telephone) and the approximate duration of the visit.

Data management and security. Research staff members collect patient information at each institution using REDCap. Each site maintains their own separate list of participant names and study ID numbers. Participants are identified on study forms and in the REDCap database by participant ID number only. To guard against the loss of confidentiality, all electronic information stored on the main database is password protected, which is also protected by antivirus software. Only research team members with appropriate training and permissions have access to the study data on shared electronic files. Data abstracted from the health record are also maintained in REDCap.

\section{Statistical analysis plan}

Statistical significance and software. All statistical tests for equivalence will be two sided with an alpha level of 0.05. We will perform all statistical analyses using STATA (version 15; STATA, College Station, TX; Computing Resource Center, Santa Monica, CA) and R software (version 3.5.3; The R Foundation, Vienna, Austria).

Intent to treat. The analyses will focus on those patients completing the study per protocol to compare telehealth PC versus in-person PC as intended without imposing assumptions about missing data. We will use the intention-to-treat principle with all randomized subjects, subsequently conducting sensitivity analyses to explore how various assumptions about missing data and differences between completers and noncompleters affect the estimated results.

If data appear to be missing at random, we will employ multiple imputation methods. However, if we find that participants do not complete the study because of clinical worsening, suggesting that data are not missing at random, we will employ pattern mixture modeling approach. We will also use the terminal decline joint modeling approach to account for missing data and compare our findings with the pattern mixture modeling approach. This approach controls for the known relationship between patient and caregiver QOL deterioration as the patient's death approaches. Based on the fitted models, we will compare participant-reported outcomes at specified times before death (one, three, and six months before the patient's death). All models will adjust for baseline criterion scores, study site, and any potential imbalances between the two groups despite randomization. The terminal decline joint modeling approach has been utilized in multiple prior PC studies given its ability to account for deterioration in outcomes closer to death. ${ }^{29}$

Sample size and power calculations. The primary endpoint of the study is to test whether telehealth PC is equivalent to in-person $\mathrm{PC}$ in the change in patient-reported QOL, as measured by the FACT-L from baseline to week 24. In our recent randomized trial of early integrated $\mathrm{PC}$ versus standard oncology care, ${ }^{4}$ we observed a $7.5(\mathrm{SD}=17.0)$ point difference in the FACT-L from baseline to week 24 favoring the early integrated PC group. Based on this, we chose a conservative equivalence margin of 4 points, which is $\sim 50 \%$ of the previously observed advantage for early integrated PC. As a 6-point difference in FACT-L score is the lowest range of a clinically meaningful difference, our equivalence margin would not reach this threshold QOL difference between telehealth and in-person PC. ${ }^{30}$ To have a $95 \%$ power to establish the equivalence of telehealth $\mathrm{PC}$ compared with in-person PC, with a two-sided alpha of 0.05 and an equivalence margin of 4 points on the FACT-L, 469 patients per group are needed. In our recent trial, ${ }^{4}$ we observed $25 \%$ missing data at 24 weeks. Therefore, we increased our planned sample size to 625 per study group.

The proposed study will also have adequate power to assess for equivalence between groups in EOL communication and hospice length of stay. Assuming the rate of EOL communication in the in-person PC group is $30 \%$, with a total sample size of 1250 , the study will have $89 \%$ power to assess for an equivalence margin of $8 \%$. For hospice length of stay, with this sample size, the study will also have $94 \%$ power to establish equivalence between groups with an equivalence margin of six days $(\mathrm{SD}=30.0)$.

Finally, this study is also adequately powered to assess for potential superiority of telehealth PC versus in-person PC in caregiver outcomes. With 313 caregivers per group, the study will have $>94 \%$ power to detect a $12 \%$ difference in caregiver participation in PC visits with telehealth compared with inperson PC (assuming the rate of caregiver participation in PC visits equals $65 \%$ based on our prior study). ${ }^{5}$ With 313 caregivers per group, we will also have $>95 \%$ power to detect a clinically meaningful difference of 3 points on the CARGOQOL $(\mathrm{SD}=10)$ and 1 point in HADS-depression scores $(\mathrm{SD}=3.0)$ between the two groups at 24 weeks, assuming that $75 \%$ of potentially eligible caregivers enroll and have a $25 \%$ missing data rate.

Evaluation of hypotheses. Aim 1: To determine whether telehealth $P C$ is equivalent to in-person $P C$ for improving patients' $Q O L$. We will use analysis of covariance (ANCOVA) models controlling for baseline criterion scores, participating site, and demographic and clinical factors (as necessary for any imbalances in baseline variables despite randomization) to assess the differences between groups in QOL at 24 weeks. We will also use linear mixed models of the longitudinal data to account for dependency among means over time and to control for study site and demographic and clinical factors (as necessary for any imbalances in baseline variables) when examining change between groups in QOL across multiple time points (i.e., baseline, weeks 12, 24, 36, and 48). We will test Heterogeneity of Treatment Effect based on age, gender, race, computer experience, and enrollment of caregivers using interaction terms in the linear mixed models. For the secondary outcomes, to compare change between groups in patient-reported depression symptoms (HADS, PHQ-9) and coping strategies (Brief Cope) from baseline to week 24 and longitudinally over time, we will again use ANCOVA and linear mixed models controlling for baseline criterion values, participating site, and demographic and clinical factors as necessary.

Aim 2: To determine whether telehealth PC is equivalent to in-person PC with respect to patient-clinician communication about EOL care preferences and length of stay in 
hospice. We will examine patients' report of discussing EOL care preference with their clinicians using the following PTPQ item: "Have you and your doctors discussed any particular wishes you have about the care you would want to receive if you were dying?" (Yes/No). We will use the final assessment either before death or at 48 weeks (whichever comes first) for this analysis. We will assess differences between study groups in the rate of patients reporting "Yes" to this item using a Fisher's exact test followed by logistic regression adjusting for study site and any imbalances in demographic and clinical factors. In addition, we will use the Wilcoxon rank sum test or ANCOVA models adjusting for study site and demographic and clinical factors as necessary to compare hospice length of stay between telehealth PC and in-person PC.

Aim 3: To compare the effect of telehealth versus inperson $P C$ on caregiver participation in $P C$ visits and outcomes. We will compare rates of caregivers' participation in PC visits between the two groups using mixed logistic models to account for correlation between multiple PC visits for each caregiver (controlling for participating site and other demographic and clinical factors as needed). In addition, we will compare change in caregiver QOL (CARGOQOL) and mood symptoms (HADS) from baseline to week 24 between the study groups using ANCOVA as described previously. We will use linear mixed models of the longitudinal data to account for dependency among means over time and to control for demographic and clinical factors (as necessary for any baseline imbalances) when examining change between groups in caregiver-reported QOL and mood across multiple time points.

To compare caregiver illness understanding (per the PTPQ) at 24 weeks between groups, we will analyze each item separately using the appropriate test (e.g., Fisher's exact test or two-sample $t$ test) and regression analyses as appropriate to adjust for study site and clinical and demographic variables. Lastly, we will compare caregivers' perception of quality of care at the EOL (per the After Death Assessment) between the two groups using ANCOVA as described previously.

Aim 4: To compare patient and caregiver satisfaction with telehealth PC versus in-person PC. We will use the final assessment of The Satisfaction and Care Delivery Questionnaire either before patient death or at 48 weeks (whichever comes first) for this analysis, comparing scores between groups using ANCOVA adjusting for study site and any imbalances in baseline factors between the two groups.

\section{Ethics determination}

The Dana-Farber/Harvard Cancer Center Internal Review Board (IRB) along with all of the other 19 participating sites' IRBs has reviewed and approved the study protocol. Each site's IRB will review all amendments to the study protocol throughout the course of the trial.

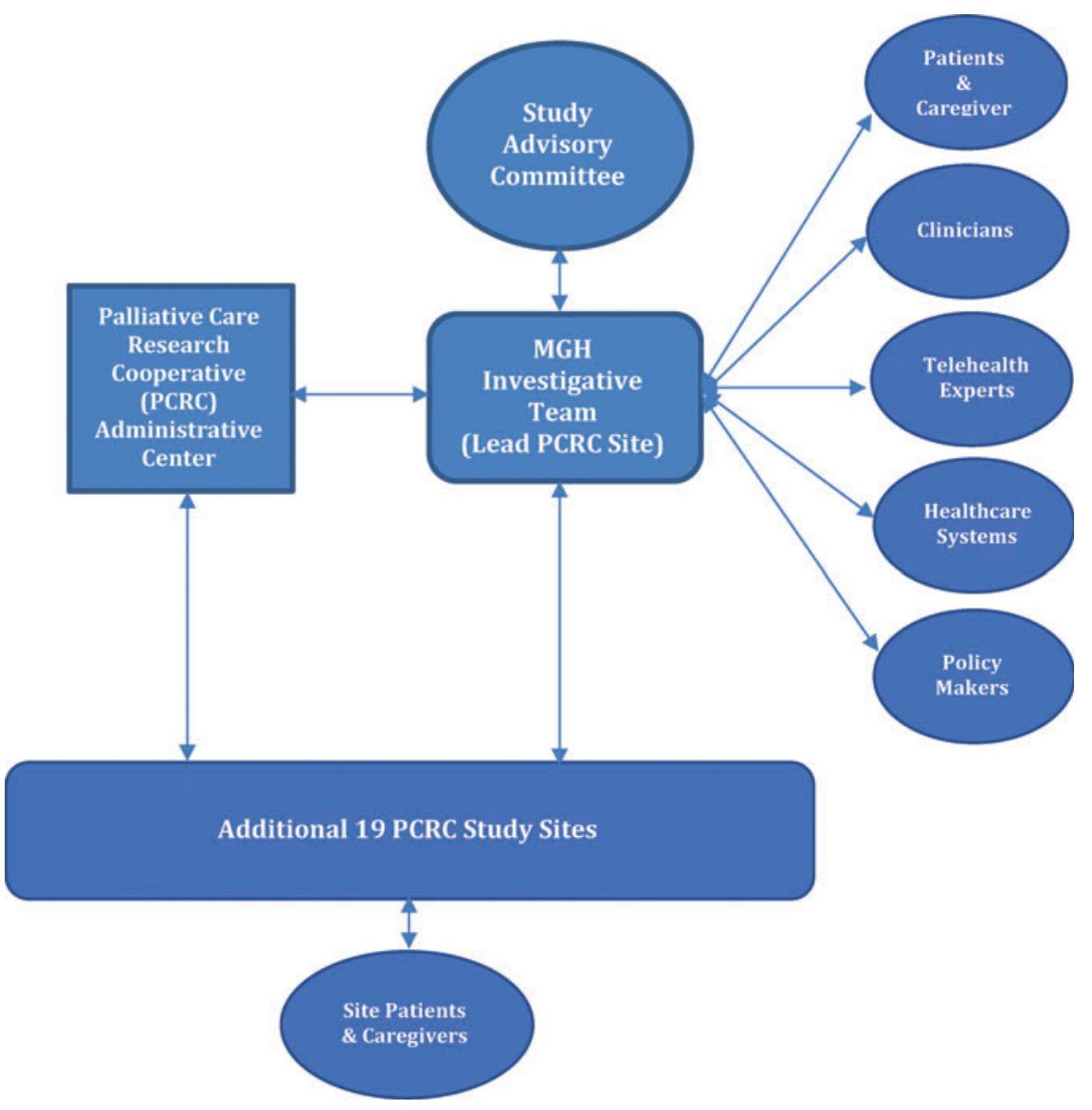

FIG. 2. Study stakeholder engagement and communication structure. 


\section{Study Implementation: Challenges and Contributions}

\section{Patient and stakeholder engagement}

We aim to ensure that this comparative effectiveness PC trial and related findings are as patient centered, meaningful, and impactful as possible by engaging and soliciting feedback from key representatives with diverse perspectives regarding all aspects of the study, including the planning, design, methods, implementation procedures, and dissemination efforts. Therefore, key patient and stakeholder partners will have significant and ongoing input throughout the trial. We have identified five core groups of patient and stakeholder partners for our proposal: (1) patients/families $(n=5),(2)$ PC and oncology clinicians $(n=4)$, (3) telehealth experts and clinician users $(n=8),(4)$ representatives from health care systems and medical insurance providers $(n=5)$, and (5) health care policy makers and advocates $(n=4)$. Also representing these constituent groups, seven additional individuals serve on the Study Advisory Committee to provide counsel to the MGH investigative team and oversight for the trial and the stakeholder engagement plan. Given that the proposed comparative effectiveness trial takes place across 20 oncology care centers, each participating institution has also identified two local, onsite patient, and caregiver partners who can provide consultation and support in conducting the study. All stakeholders receive $\$ 100$ per hour as remuneration for their time in collaborating and assisting with the study. Figure 2 and Table 3 depict the structure, roles, and activities of the stakeholder groups.

To ensure mutual learning between the research team and patient and stakeholder partners, we employ rigorous methods for collecting and communicating information. During each phase of the study, the MGH research team will facilitate the stakeholder group interviews, compile all stakeholder recommendations, and review them point-by-point with the investigative team, modifying the protocol based on consensus. To ensure clear communication and transparency, the research team will e-mail all stakeholders twice annually a

Table 3. Stakeholder Roles and Activities Throughout Study

\begin{tabular}{|c|c|c|c|}
\hline Meeting/activity & Frequency & Setting/method & Purpose \\
\hline $\begin{array}{l}\text { Study Advisory } \\
\text { Committee }\end{array}$ & $\begin{array}{l}\text { Three meetings } \\
\text { annually } \\
\text { throughout the } \\
\text { study }\end{array}$ & $\begin{array}{l}\text { Two meetings through } \\
\text { teleconference call } \\
\text { and one in-person } \\
\text { meeting at MGH } \\
\text { per year }\end{array}$ & $\begin{array}{l}\text { - Year 1: Provide feedback on protocol } \\
\text { procedures, outcome measures, trial start- } \\
\text { up, stakeholder engagement plan } \\
\text { - Years 2-4: Review study progress } \\
\text { including patient recruitment and retention, } \\
\text { monitor data safety procedures, problem- } \\
\text { solve with the MGH team any study } \\
\text { implementation concerns, review } \\
\text { stakeholder engagement activities } \\
\text { - Year 5: Review preliminary study findings } \\
\text { and discuss dissemination plans }\end{array}$ \\
\hline $\begin{array}{l}\text { Five stakeholder group } \\
\text { meetings } \\
\text { - Patients and caregivers } \\
\text { - PC and oncology } \\
\text { clinicians/researchers } \\
\text { - Telehealth experts } \\
\text { - Health care system } \\
\text { representatives and } \\
\text { insurers } \\
\text { - Policy makers and } \\
\text { advocates }\end{array}$ & $\begin{array}{l}\text { Two meetings } \\
\text { annually } \\
\text { throughout study }\end{array}$ & $\begin{array}{l}\text { Meetings will occur } \\
\text { through } \\
\text { teleconference }\end{array}$ & $\begin{array}{l}\text { - Year 1: Provide feedback on study design, } \\
\text { aims, protocol procedures, outcome } \\
\text { measures, recruitment methods } \\
\text { - Years 2-4: Review study progress, } \\
\text { recruitment, and retention; obtain feedback } \\
\text { to problem-solve any challenges with } \\
\text { implementing study; discuss any } \\
\text { innovations in field with respect to PC and/ } \\
\text { or telehealth programs and how might } \\
\text { impact study } \\
\text { - Year 5: Review preliminary study findings } \\
\text { and discuss dissemination plans }\end{array}$ \\
\hline $\begin{array}{l}\text { Local site patients and } \\
\text { caregiver stakeholder } \\
\text { meetings }\end{array}$ & $\begin{array}{l}\text { At least two } \\
\text { meetings annually } \\
\text { throughout study }\end{array}$ & $\begin{array}{l}\text { Meetings will occur in } \\
\text { person at each of } \\
\text { the study site } \\
\text { institutions }\end{array}$ & $\begin{array}{l}\text { - Year 1: Review the study protocol and } \\
\text { obtain feedback on any site-specific } \\
\text { recommendations for ensuring successful } \\
\text { study implementation; assist with } \\
\text { developing site-specific strategies for } \\
\text { optimizing participant recruitment and } \\
\text { retention } \\
\text { - Years 2-4: Review study progress and } \\
\text { obtain advice and support throughout the } \\
\text { study to problem-solve any challenges in } \\
\text { implementing study procedures } \\
\text { - Year 5: Identify avenues for disseminating } \\
\text { the study results through local health care } \\
\text { organizations, media outlets, and patient } \\
\text { groups }\end{array}$ \\
\hline
\end{tabular}


summary of the feedback obtained from all group meetings with a description of the ways the protocol was modified in response. Similarly, during each phase of the study, each site's lead investigator will provide a written summary of the feedback they receive from their onsite patient and caregiver stakeholders, which the MGH research team will collate and deliver back to all sites to facilitate community learning.

\section{Fidelity monitoring in the context of complex interventions}

For fidelity monitoring, we use rigorous research methods that are adherent to the PCORI Methodology Standards and include a strong theoretical foundation, a comprehensive training approach, and a systematic assessment of intervention delivery. Table 4 details the steps the MGH research team and site investigators are taking to ensure the fidelity of theory and design, training, and intervention delivery.

\section{Response to challenges and barriers to study success}

At the time of this submission, the first year of the study has completed. To date, all study sites have executed their research agreements with $\mathrm{MGH}$; obtained IRB approval at their respective institutions; completed comprehensive training in study procedures, use of telehealth, and implementation of the PC intervention; and begun recruitment and enrollment of patients and their caregivers.

The primary challenges and barriers to study success include institutional, clinician, and patient factors. At the institutional level, integration of a new telehealth platform into existing electronic systems for scheduling and billing for patient visits may be limited. In addition, infrastructure and resources are often lacking to identify eligible patients either through electronic reports or through staff screening. Although early PC has become the recommended standard of care based on robust evidence from clinical trials, some

Table 4. Procedures for Ensuring Fidelity of Study Design, Methods, and Intervention Delivery

\begin{tabular}{ll}
\hline Types of fidelity & \multicolumn{1}{c}{ Procedures to ensure fidelity } \\
\hline Theory and design & - Intervention development based on a well- \\
& defined conceptual model and systematic \\
review of literature & - Standardization of intervention dose with clear \\
& feasibility data based on prior work \\
& Minimization of cross-contamination effects \\
& given both groups will receive PC
\end{tabular}

Training

Intervention delivery by PC clinicians
- Development of comprehensive guides and standard operating procedures for site PIs, clinical research coordinators, and PC clinicians

- Initial in-person training of lead site investigators and PC clinicians on PC intervention and use of telehealth

- Initial in-person training of site research coordinators on study procedures and use of telehealth

- Onsite training at each participating site with all PC clinicians providing care to study patients

- Monthly conference calls (led by MGH team) with site investigators to address study issues

- Monthly conference calls (led by MGH team) with research coordinators to address study procedures

- Annual retraining seminar through videoconferencing with lead site investigators and $\mathrm{PC}$ clinicians

- Utilization of PC intervention guide with standardized content areas

- Completion of electronic survey after each study visit to record the content and topics that the PC clinician addressed

- Documentation of clinical encounter using a standardized visit note template in site health record
Fidelity assessment

- Utilize evidence-based PC intervention guide based on multiple prior trials

- Measure number of PC intervention visits and visit duration using electronic PC clinician encounter survey

- Measure number of in-person PC visits in the telehealth group and telephone visits in the inperson group

- Complete review of site investigator, research coordinator, and PC intervention guides and training videos

- Complete training of all site investigators and lead PC clinicians in protocol administration and intervention delivery

- Complete training of all site research coordinators on study procedures, protocol implementation, and use of technology

- Assess pre- and postknowledge that PC clinicians and research coordinators acquired during training

- Send meeting minutes from conference calls to all site investigators and PC clinicians

- Send meeting minutes from conference calls to all site research coordinators

- Assess pre- and postknowledge that lead site investigators and PC clinicians acquired during retraining

- Conduct ongoing training of any new staff in standardized PC intervention guide/videos

- Review PC electronic surveys quarterly to ensure adherence to content (by MGH team). These findings will be discussed during monthly meetings with lead site investigators

- Review a random sample of PC visit notes quarterly to ensure adherence to content (by lead site investigators)

PIs, principal investigators. 
oncologists may still be unwilling or uncomfortable to refer their newly diagnosed patients with advanced lung cancer for such care or engage in joint visits with PC clinicians. Alternatively, maintaining motivation and engagement with $\mathrm{PC}$ clinicians may be challenging over the course of the study, especially when they are often donating time to schedule and meet with study patients and complete visit surveys. For those patients assigned to in-person PC, the scheduling of visits on the same day between oncology and PC requires considerable coordination. Also, patient barriers include reluctance or discomfort with using telehealth technology, misperceptions about the role of early PC, and feeling overwhelmed by being approached for multiple studies.

To address these challenges, the MGH research team created print and video-based educational materials and conducted extensive in-person and remote training with study site investigators, PC clinicians, and clinical research coordinators on effective study procedures. For example, much of the training has focused on identifying and collaborating with thoracic oncologist champions for the study; standardizing methods for identifying all eligible patients to approach for enrollment; employing scripts with optimal language when introducing PC to patients and families; working closely with the PC scheduler to coordinate study visits within the flow of oncology care; and enhancing PC clinician and patient comfort with telehealth technology by conducting initial test training calls and providing recommendations for PC clinicians to establish rapport while using videoconferencing. In addition, we have expanded the patient eligibility period for enrollment from 8 to 12 weeks from diagnosis, increasing opportunities for recruitment. To overcome oncologist reluctance to engage with PC, we have encouraged the PC clinicians to conduct joint visits with oncologists to observe one another's practice and develop a collaborative partnership. Many of the research assistants have also practiced mock enrollments with site stakeholders and shadowed the PC clinicians to enhance their understanding of PC so that they can communicate effectively with patients and families about the role and benefits of the intervention. Finally, to ensure successful implementation of the trial and problem-solve any challenges that arise, the MGH research team has begun conducting monthly videoconference calls with small groups of three to four sites, including the lead investigators and PC clinicians, to review

Table 5. Dissemination Plan and Channels

\begin{tabular}{|c|c|c|}
\hline Intended audience & Collaborative parties & Communication channel \\
\hline $\begin{array}{l}\text { General public and health } \\
\text { care communities }\end{array}$ & $\begin{array}{l}\text { Site investigator(s) with their } \\
\text { respective media and public affairs } \\
\text { office }\end{array}$ & $\begin{array}{l}\text { News outlets through: } \\
\text { Newspapers (e.g., USA Today) } \\
\text { Social Media (e.g., Twitter, Facebook, and } \\
\text { LinkedIn) } \\
\text { News Shows (e.g., NPR) }\end{array}$ \\
\hline Oncology and PC community & Site investigators and staff & $\begin{array}{l}\text { Articles in high-profile peer review publications } \\
\text { Presentations at national meetings (e.g., } \\
\text { American Society of Clinical Oncology and } \\
\text { the American Academy of Hospice and } \\
\text { Palliative Medicine) } \\
\text { Advocacy organizations (e.g., Center to } \\
\text { Advance Palliative Care) }\end{array}$ \\
\hline $\begin{array}{l}\text { Policy makers and leaders of } \\
\text { health care institutions }\end{array}$ & $\begin{array}{l}\text { Lead investigators with Dr. Lee } \\
\text { Schwamm } \\
\text { - Health care organizations (e.g., } \\
\text { ASCO, ACS, and CAPC) }\end{array}$ & $\begin{array}{l}\text { Articles in policy journals (e.g., Health Affairs) } \\
\text { Advocacy for palliative telehealth } \\
\text { reimbursement and to increase the number of } \\
\text { trained PC clinicians }\end{array}$ \\
\hline Local health care systems & $\begin{array}{l}\text { Site study team and clinicians along } \\
\text { with patient and caregiver } \\
\text { stakeholders }\end{array}$ & $\begin{array}{l}\text { Each site will, respectively, present study } \\
\text { findings to their institutions in collaboration } \\
\text { with their patient and caregiver stakeholders. } \\
\text { In addition to normal forums like grand } \\
\text { rounds, the teams will reach out to local } \\
\text { hospitals and advocacy organizations along } \\
\text { with a presentation to the patient and family } \\
\text { advisory council. }\end{array}$ \\
\hline $\begin{array}{l}\text { Patients and families affected } \\
\text { by serious illness } \\
\text { Health care providers and } \\
\text { systems }\end{array}$ & $\begin{array}{l}\text { - Lead investigators } \\
\text { - MGH and partners health care } \\
\text { stakeholders } \\
\text { - Five key health care insurers }\end{array}$ & $\begin{array}{l}\text { Work with insurers to develop reimbursement } \\
\text { models specifically for PC delivered by } \\
\text { telehealth and creating new capacity for this } \\
\text { delivery model }\end{array}$ \\
\hline $\begin{array}{l}\text { Health care teams } \\
\text { Patients and caregivers }\end{array}$ & Lead investigators and study teams & $\begin{array}{l}\text { Publish and present on the training methodology } \\
\text { for patients, caregivers, clinicians, and } \\
\text { training staff to leverage the telehealth video } \\
\text { platform for care delivery. }\end{array}$ \\
\hline Study patients and caregivers & MGH lead site & E-mail and direct mail all study results \\
\hline
\end{tabular}

ACS, American Cancer Society; ASCO, American Society of Clinical Oncology; CAPC, Center to Advance Palliative Care. 
study procedures, recruitment efforts, data collection, and intervention delivery.

The challenges of the trial to date are consistent with findings from a systematic review of studies on this use of video consultations in PC. In this review, several themes emerged with respect to the advantages and disadvantages of using videoconferencing in PC including the redesign of care; communication among patients, family members, and the clinicians; users' perceptions of video consultations; technology issues; privacy concerns; and economic considerations. ${ }^{14}$ As our study progresses, other potential challenges may include how PC clinicians address sensitive topics with patients and families through telehealth, including discussions about preferences for EOL care and advance care planning. Although some research is beginning to take place on telephonic discussions of advance care planning, ${ }^{31}$ further study is needed to discern the best practices for such conversations using videoconferencing to ensure patients and families have accurate understanding of their illness and sufficient support to communicate their goals and wishes.

\section{Plans for dissemination and spread}

Our study is of significant importance to: (1) determining the applicability of telehealth in caring for patients with advanced cancer, (2) deepening our understanding of whether utilizing telehealth increases the effective reach of PC for diverse patients and families in community settings, and (3) improving the care delivery system for many stakeholders including patients, caregivers, clinicians, health care institutions, insurers, and technology developers. Therefore, we are planning to disseminate results broadly through several communication channels (Table 5).

Each participating site will engage their respective media teams and public affair offices, work with their patient and caregiver stakeholders, and collaborate with the lead site (MGH) to communicate the results through various news outlets, articles, presentations both local and national, and advocacy organizations. Additional materials will be developed for patients, caregivers, and health care teams to leverage the telehealth modality to extend access to clinical care. The multicenter research group collectively will also communicate all study findings to all patients and caregivers who participated in the study.

The MGH research team will spearhead opportunities to impact health care policy and reimbursement models. One of our coinvestigators is Dr. Lee Schwamm, who has published a number of key articles on implementing telehealth platforms, including "Telehealth: seven strategies to successfully implement disruptive technology and transform health care." 32 The lead investigators will work with Dr. Schwamm to publish findings in Health Affairs with the intention of impacting health care policy. In addition, MGH and Partners Healthcare leadership, along with the lead investigators, will work with a number of insurance companies to develop appropriate reimbursement models to compensate for the clinical care provided to patients and families through telehealth.

Finally, there will be an active social media campaign to amplify the findings and implications. Platforms to be included are Twitter, Facebook, Instagram, and LinkedIn, some of the most popular sites for such purposes. This effort will include the establishment of an official Twitter account for the study, a Facebook page for the study, and an Instagram account, which will be monitored and content delivered by a member of the research team under the oversight of the principal investigators (PIs). Members of the investigative team who are most active on social media or are members of key committees on national organizations will both personally amplify the messages and train other members of the team in the effective use of social media. Such approaches will include the creation of visual content with graphically appealing tables/figure, photographs of investigators, Tweetchats of key findings, and visual abstracts from conference presentations.

\section{Author Disclosure Statement}

All statements in this report, including its findings and conclusions, are solely those of the authors and do not necessarily represent the views of the Patient-Centered Outcomes Research Institute (PCORI), its Board of Governors, or Methodology Committee.

This is a study protocol and thus does not contain or reflect any primary data.

This work is supported through a Patient-Centered Outcomes Research Institute (PCORI) Award (PLC-160935995) and by the Palliative Care Research Cooperative Group funded by the National Institute of Nursing Research U2CNR014637.

No competing financial interests exist.

\section{References}

1. Zimmerman C, Riechelmann R, Krazanowska M, et al.: Effectiveness of specialized palliative care a systematic review. JAMA 2008;299:1698-1709.

2. Zimmermann C, Swami N, Krzyzanowska M, et al.: Early palliative care for patients with advanced cancer: A clusterrandomised controlled trial. Lancet 2014;383:1721-1730.

3. Temel JS, Greer JA, Muzikansky A, et al.: Early palliative care for patients with metastatic non-small-cell lung cancer. N Engl J Med 2010;363:733-742.

4. Temel JS, Greer JA, El-Jawahri A, et al.: Effects of early integrated palliative care in patients with lung and GI cancer: A randomized clinical trial. J Clin Oncol 2017;35:834-841.

5. El-Jawahri A, Greer JA, Pirl WF, et al.: Effects of early integrated palliative care on caregivers of patients with lung and gastrointestinal cancer: A randomized clinical trial. Oncologist 2017;22:1528-1534.

6. Ferrell BR, Temel JS, Temin S, et al.: Integration of palliative care into standard oncology care: American Society of Clinical Oncology clinical practice guideline update. J Clin Oncol 2017;35:96-112.

7. Lupu D, American Academy of H, Palliative Medicine Workforce Task F: Estimate of current hospice and palliative medicine physician workforce shortage. J Pain Symptom Manage 2010;40:899-911.

8. Spetz J, Dudley N, Trupin L, et al.: Few hospital palliative care programs meet national staffing recommendations. Health Affairs 2016;35:1690-1697.

9. Hui D, Elsayem A, De La Cruz M, et al.: Availability and integration of palliative care at US cancer centers. JAMA 2010;303:1054-1061.

10. What is Telehealth? https://catalyst.nejm.org/what-istelehealth. (Last accessed January 31, 2019). 
11. Shigekawa E, Fix M, Corbett G, et al.: The current state of telehealth evidence: A rapid review. Health Affairs 2018; 37:1975-1982.

12. Marcin J, Rimsza M, Moskowitz W: The use of telemedicine to address access and physician workforce shortages. Pediatrics 2015;136:202-209.

13. Porter LS, Samsa G, Steel JL, et al.: Caregiver-guided pain coping skills training for patients with advanced cancer: Background, design, and challenges for the CaringPals study. Clin Trials 2019;16:263-272.

14. Jess M, Timm H, Dieperink KB: Video consultations in palliative care: A systematic integrative review. Palliat Med 2019 [Epub ahead of print]; DOI: 10.1177/ 0269216319854938.

15. Bakitas M, Lyons KD, Hegel MT, et al.: Effects of a palliative care intervention on clinical outcomes in patients with advanced cancer: The Project ENABLE II randomized controlled trial. JAMA 2009;302:741-749.

16. Dionne-Odom JN, Azuero A, Lyons KD, et al.: Benefits of early versus delayed palliative care to informal family caregivers of patients with advanced cancer: Outcomes from the ENABLE III randomized controlled trial. J Clin Oncol 2015;33:1446-1452.

17. Yoong J, Park ER, Greer JA, et al.: Early palliative care in advanced lung cancer: A qualitative study. JAMA Intern Med 2013;173:283-290.

18. Hoerger M, Greer JA, Jackson VA, et al.: Defining the elements of early palliative care that are associated with patient-reported outcomes and the delivery of end-of-life care. J Clin Oncol 2018;36:1096-1102.

19. Cella D: The Functional Assessment of Cancer TherapyLung and Lung Cancer Subscale assess quality of life and meaningful symptom improvement in lung cancer. Semin Oncol 2004;31(3 Suppl 9):11-15.

20. Wright AA, Zhang B, Ray A, et al.: Associations between end-of-life discussions, patient mental health, medical care near death, and caregiver bereavement adjustment. JAMA 2008;300:1665-1673.

21. El-Jawahri A, Traeger L, Park ER, et al.: Associations among prognostic understanding, quality of life, and mood in patients with advanced cancer. Cancer 2014;120:278285.

22. Rubin HR, Gandek B, Rogers WH, et al.: Patients' ratings of outpatient visits in different practice settings. Results from the Medical Outcomes Study. JAMA 1993;270:835840.

23. Carver CS: You want to measure coping but your protocol's too long: Consider the brief COPE. Int J Behav Med 1997;4:92-100.

24. Hagan TL, Fishbein JN, Nipp RD, et al.: Coping in patients with incurable lung and gastrointestinal cancers: A validation study of the brief COPE. J Pain Symptom Manage 2017;53:131-138.

25. Minaya P, Baumstarck K, Berbis J, et al.: The CareGiver Oncology Quality of Life questionnaire (CarGOQoL): Development and validation of an instrument to measure the quality of life of the caregivers of patients with cancer. Eur J Cancer 2012;48:904-911.

26. Zigmond AS, Snaith RP: The hospital anxiety and depression scale. Acta Psychiatr Scand 1983;67:361-370.

27. Kroenke K, Spitzer RL, Williams JB: The PHQ-9: Validity of a brief depression severity measure. J Gen Intern Med 2001;16:606-613.

28. Sangha O, Stucki G, Liang $\mathrm{MH}$, et al.: The SelfAdministered Comorbidity Questionnaire: A new method to assess comorbidity for clinical and health services research. Arthritis Rheum 2003;49:156-163.

29. Li Z, Tosteson TD, Bakitas MA: Joint modeling quality of life and survival using a terminal decline model in palliative care studies. Stat Med 2013;32:1394-1406.

30. Cella D, Hahn EA, Dineen K: Meaningful change in cancerspecific quality of life scores: Differences between improvement and worsening. Qual Life Res 2002;11:207-221.

31. Boettcher I, Turner R, Briggs L: Telephonic advance care planning facilitated by health plan case managers. Palliat Support Care 2015;13:795-800.

32. Schwamm LH: Telehealth: Seven strategies to successfully implement disruptive technology and transform health care. Health Affairs 2014;33:200-206.

Address correspondence to:

Joseph A. Greer, PhD

Massachusetts General Hospital

Yawkey Center

55 Fruit Street, Suite $10 B$

Boston, MA 02114

E-mail: jgreer2@mgh.harvard.edu 